PHYSICAL REVIEW D 88, 055009 (2013)

\title{
Phenomenology of heavy vectorlike leptons
}

\author{
Koji Ishiwata and Mark B. Wise \\ California Institute of Technology, Pasadena, California 91125, USA
}

(Received 19 July 2013; published 9 September 2013)

\begin{abstract}
We study the impact that a heavy generation of vectorlike leptons can have on the value of the electric dipole moment of the electron, and the rates for the flavor violating processes $\mu \rightarrow e \gamma$ and $\mu \rightarrow 3 e$. The smallness of the charged lepton masses suggests that at least some of the Yukawa coupling constants of the vectorlike leptons to the ordinary leptons or amongst themselves are small, but even with such small couplings experiments trying to detect these quantities are sensitive to extra generation lepton masses up to about $100 \mathrm{TeV}$.
\end{abstract}

DOI: 10.1103/PhysRevD.88.055009

PACS numbers: $12.60 . \mathrm{Cn}, 12.60 .-\mathrm{i}$

\section{INTRODUCTION}

We have experimental evidence for three generations of quarks and leptons. There are powerful constraints on the possibility of an additional standard model like chiral generation of quarks and leptons from LHC data. These come mostly from the constraints on the additional quarks. At present we do not have a good understanding of why there are three generations and so it is worth exploring the possible experimental consequences of additional fermions. One possibility is an additional vectorlike generation, where a standard model-like generation is paired with one of opposite chirality. For such fermions mass terms are allowed and so the masses of such fermions could be much larger than the electroweak (EW) scale, and furthermore the masses of the quarks could be very different from those of the leptons. For any value of the vectorlike fermion masses, adding these particles is technically natural since there are no quadratically divergent contributions to their masses. In this paper we explore some of the experimental consequences of vectorlike leptons (assuming either the vectorlike quarks are much heavier than the leptons or do not exist).

Vectorlike leptons have already been studied extensively in the literature. They have been added to cancel anomalies in models that extend the gauge group to include $U(1)$ lepton number and possibly also baryon number (see, for example, [1]). We considered the impact of vectorlike leptons on the properties of the Higgs boson under the assumption that almost all of their masses come from their couplings to the Higgs boson [2]. In Ref. [3], the contribution of additional leptons to muon $g-2$ was studied. They considered several models, which consist of extra leptons with different EW charges, and derived the Wilson coefficient for $\bar{\mu} \sigma^{\mu \nu} \mu F_{\mu \nu}$. Here $F_{\mu \nu}$ is the field strength of the electromagnetic field. Bounds from precision data were also discussed because the extra leptons induce dimension six operators, such as $\left(H^{\dagger} D_{\mu} \tau^{a} H\right)\left(\bar{L} \gamma^{\mu} \tau^{a} L\right)$, $\left(H^{\dagger} D_{\mu} H\right)\left(\bar{L} \gamma^{\mu} L\right)$, and $\left(H^{\dagger} D_{\mu} H\right)\left(\bar{e}_{R} \gamma^{\mu} e_{R}\right)$. Here $H=$ $\left(H^{+}, H^{0}\right)^{T}$ is the Higgs doublet, $L=\left(\nu_{L}, e_{L}\right)^{T}$, and $e_{R}$ are the lepton doublets and singlets (flavor indices are omitted), $\tau^{a}$ is a Pauli matrix, and $D_{\mu}$ denotes the covariant derivative. Constraints from precision EW measurements were also discussed in Refs. [4-6]. In Refs. [5,6], the impact of additional vectorlike matter on $h \rightarrow \tau \tau, b b$, $\gamma \gamma$ was studied. The impact on $h \rightarrow \mu \mu$ and the renormalization group equations for the Higgs quartic coupling, gauge couplings, and production rate of the Higgs boson at the LHC, were also studied. Motivated by the deviation of muon magnetic moment from the standard model prediction, Ref. [7] studied phenomenology of Higgs decay at the LHC, as well as the running of Higgs quartic coupling and gauge couplings. In Ref. [8], flavor violating processes $(\tau \rightarrow \mu \gamma, \tau \rightarrow e \gamma, \mu \rightarrow e \gamma, \tau \rightarrow 3 \mu, \tau \rightarrow 3 e, \mu \rightarrow 3 e)$, magnetic and electric dipole moments, $\mu \rightarrow e$ conversion, and flavor violating Higgs decay were studied from the effective theory point of view. They carefully pointed out regions of parameter space which avoid a tuning of lepton Yukawa couplings. See also Ref. [9] which studied Higgs to dilepton decay and $C P$ and lepton flavor violating process.

In this paper we focus on weakly coupled vectorlike leptons in the region of parameter space where they are heavy, i.e., masses significantly above one TeV. Although they change Higgs properties by a very small amount and are not constrained by precision EW measurement, we find that vectorlike leptons with masses up to about $100 \mathrm{TeV}$ can contribute at the present experimental limit to lepton flavor violating muon decay and the electric dipole moment of the electron without unnatural assignments for their Yukawa coupling constants. Even though our calculation of the amplitude for $\mu \rightarrow e \gamma$ exists in the literature (see Refs. $[3,7,10,11]$ ) and its enhancement due to mass mixing of the vectorlike fermions has been noted previously, we do address a few new issues including the tree-level decay rate for $\mu \rightarrow 3 e$, the electric dipole moment of the electron, and the one-loop correction to mass matrix for the light leptons. Furthermore our focus on the multi- $\mathrm{TeV}$ regime is different from most of the previous literature and this allows for simplification of the formulas for dipole moments and the amplitudes for flavor violating decays of charged leptons. 
One goal of this paper is to explore whether the enhancement in the amplitudes for the chirality flipping radiative processes $\mu \rightarrow e \gamma$ and the electric dipole moment of the electron, arising from heavy vectorlike lepton mixing, results in sensitivity to much higher mass scales for the new vectorlike leptons. We find that this is not the case if naturalness in the mass matrix for the standard model charged leptons is imposed. ${ }^{1}$ We also stress that for equal branching ratios $\mu \rightarrow 3 e$ is sensitive to higher masses for the vectorlike leptons than $\mu \rightarrow e \gamma$.

\section{THE MODEL}

We add to the standard model a fourth generation of leptons, which have the same quantum numbers under the gauge group as the standard model leptons. We call them $L_{L}^{\prime}, e_{R}^{\prime}$ and $L_{R}^{\prime \prime}, e_{L}^{\prime \prime}$ where $L_{L}^{\prime}=\left(\nu_{L}^{\prime}, e_{L}^{\prime}\right)^{T}$ and $L_{R}^{\prime \prime}=$ $\left(\nu_{R}^{\prime \prime}, e_{R}^{\prime \prime}\right)^{T}$. Here $L_{L}^{\prime}, e_{R}^{\prime}$ is another copy of a standard model lepton generation, while $L_{R}^{\prime \prime}, e_{L}^{\prime \prime}$ is the one with the opposite chirality. (A similar model is discussed in Refs. [3-7].) Hence there is no gauge anomaly caused by these extra leptons. The new terms in the Lagrangian density that involve these additional leptons are

$$
\begin{aligned}
\mathcal{L}= & -M_{L} \bar{L}_{L}^{\prime} L_{R}^{\prime \prime}-M_{E} \bar{e}_{R}^{\prime} e_{L}^{\prime \prime}-h_{E}^{\prime} \bar{L}_{L}^{\prime} H e_{R}^{\prime}-h_{E}^{\prime \prime} \bar{L}_{R}^{\prime \prime} H e_{L}^{\prime \prime} \\
& -\lambda_{E}^{i} \bar{L}_{L}^{\prime} H e_{R}^{i}-\lambda_{L}^{i} \bar{L}_{L}^{i} H e_{R}^{\prime}+\text { H.c. }
\end{aligned}
$$

$L_{L}^{i}, e_{R}^{i}(i=e, \mu, \tau)$ are mostly the standard model leptons. In addition there are the gauge invariant kinetic terms involving these leptons. We have chosen a basis where terms such as $\bar{L}_{R}^{\prime \prime} L_{L}^{i}$ and $\bar{e}_{L}^{\prime \prime} e_{R}^{i}$ are rotated away. This fact is mentioned in Ref. [6]. In that basis we write the Yukawa interactions of the ordinary generations as

$$
\mathcal{L}_{Y}=-Y_{e}^{i j} \bar{L}_{L}^{i} H e_{R}^{j}+\text { H.c. }
$$

Introducing the four component fields, $e_{1}=\left(e_{L}^{\prime}, e_{R}^{\prime \prime}\right)^{T}$, $e_{2}=\left(e_{L}^{\prime \prime}, e_{R}^{\prime}\right)^{T}$, and $\nu_{1}=\left(\nu_{L}^{\prime}, \nu_{R}^{\prime \prime}\right)^{T}$, the Lagrangian density becomes

$$
\begin{aligned}
\mathcal{L}= & -M_{L} \bar{e}_{1 L} e_{1 R}-M_{L} \bar{\nu}_{1 L} \nu_{1 R}-M_{E} \bar{e}_{2 R} e_{2 L} \\
& -h_{E}^{\prime}\left(H^{0} \bar{e}_{1 L}+H^{+} \bar{\nu}_{1 L}\right) e_{2 R}-h_{E}^{\prime \prime}\left(H^{0} \bar{e}_{1 R}+H^{+} \bar{\nu}_{1 R}\right) e_{2 L} \\
& -\lambda_{E}^{i}\left(H^{0} \bar{e}_{1 L}+H^{+} \bar{\nu}_{1 L}\right) e_{R}^{i}-\lambda_{L}^{i} H^{0} \bar{e}_{L}^{i} e_{2 R}+\text { H.c. }
\end{aligned}
$$

We are primarily interested in the processes $\mu \rightarrow e \gamma$, $\mu \rightarrow 3 e$ and the electric dipole moment (EDM) of the electron. One reason is that the experimental bounds for the processes are very stringent, i.e., the branching fraction for $\mu \rightarrow e \gamma$ and $\mu \rightarrow 3 e$ should satisfy $[13,14]$

$$
\operatorname{Br}(\mu \rightarrow e \gamma)<2.4 \times 10^{-12}
$$

\footnotetext{
${ }^{1} \mathrm{~A}$ similar situation occurs with lepton flavor violation induced at one loop by scalar leptoquarks; see Ref. [12].
}

$$
\operatorname{Br}(\mu \rightarrow 3 e)<1.0 \times 10^{-12},
$$

respectively, while the EDM of the electron is very suppressed [15]

$$
\left|d_{e}\right|<1.05 \times 10^{-27} e \mathrm{~cm} \text {. }
$$

Another important reason is that the chirality flip in the radiative processes (i.e., $\mu \rightarrow e \gamma$ and electron EDM) is not suppressed by the masses of the muon and electron respectively since mass mixing of the heavy leptons in a loop diagram can cause the chirality flip. Regarding $\mu \rightarrow 3 e$, we will see that this process occurs at tree level, which enhances its importance compared with the loop process $\mu \rightarrow e \gamma$. This is only partially degraded by the phase space suppression associated with the additional particle in the final state.

The standard model leptons are quite light and so if there are no awkward cancellations in their mass matrices one expects hierarchical dimensionless standard model Yukawa couplings $Y_{e}^{i j}$ in the range, $10^{-2}-10^{-5}$. Naively one might expect that the same is true for both the couplings $\lambda_{E}^{i}$ and $\lambda_{L}^{i}$ since the fields $L_{L}^{\prime}$ and $e_{R}^{\prime}$ have the same quantum numbers as the standard model fields. As will be shown later, the parts of the amplitudes for $\mu \rightarrow e \gamma$ and electron EDM that are not suppressed by the muon or electron mass are proportional to the product of dimensionless couplings, $\epsilon=\lambda_{E} \lambda_{L} h_{E}^{\prime \prime \prime \prime}$. (Here the flavor indices of $\lambda_{L, E}^{i}$ are omitted.) Hence, the above argument would give rise to the expectation that $\epsilon$ is very small, i.e., in the range $10^{-4}-10^{-10}$. However this is not necessarily the case. To make the argument quantitative we now show that it is possible to find a global $U(1)$ symmetry that forces the standard model Yukawa coupling constants $Y_{e}^{i j}$ to vanish but forces only one of the couplings in the product $\epsilon$ to vanish. Under this symmetry the fields transform as $L_{L}^{i} \rightarrow e^{i q_{L} \alpha} L_{L}^{i}, e_{R}^{i} \rightarrow e^{i q_{e} \alpha} e_{R}^{i}, e_{1} \rightarrow e^{i q_{1} \alpha} e_{1}$, and $e_{2} \rightarrow e^{i q_{2} \alpha} e_{2}$. We take $q_{e} \neq q_{L}$ to forbid the standard model Yukawa interactions. Then it is not possible to choose the charges $q_{1}$ and $q_{2}$ so all the coupling constants $\lambda_{E}^{i}, \lambda_{L}^{i}$, and $h_{E}^{\prime, I I}$ are nonzero. But it is possible to choose values of the charges so only one of these couplings is zero,

(i) $q_{1}=q_{e}, q_{2}=q_{L}$ forces $h_{E}^{\prime}=h_{E}^{\prime \prime}=0$ but allows nonzero values for $\lambda_{E}^{i}$ and $\lambda_{L}^{i}$.

(ii) $q_{1}=q_{2}=q_{L}$ forces $\lambda_{E}^{i}=0$ but allows nonzero values for $\lambda_{L}^{i}$ and $h_{E}^{\prime, \prime \prime}$.

(iii) $q_{1}=q_{2}=q_{e}$ forces $\lambda_{L}^{i}=0$ but allows nonzero values for $\lambda_{E}^{i}$ and $h_{E}^{\prime, \prime \prime}$.

Given these results, it is perfectly reasonable to consider the product of couplings $\epsilon$ in the range $10^{-2}-10^{-5}$ and perhaps even larger if one takes a different point of view than we have adopted.

We now transition to the mass eigenstate basis for the charged lepton fields which we denote by a tilde. The $5 \times 5$ charged lepton mass matrix $\mathcal{M}$ is defined by 


$$
\begin{aligned}
\mathcal{L}_{\text {mass }} & =-\left(\begin{array}{lll}
\bar{e}_{1} & \bar{e}_{2} & \bar{e}^{i}
\end{array}\right)_{L}\left(\begin{array}{ccc}
M_{L} & m^{\prime} & \mu_{E}^{i} \\
m^{\prime * *} & M_{E} & 0_{1 \times 3} \\
0_{3 \times 1} & \mu_{L}^{i} & 0_{3 \times 3}
\end{array}\right)\left(\begin{array}{c}
e_{1} \\
e_{2} \\
e^{i}
\end{array}\right)_{R}+\text { H.c. } \\
& \equiv-\bar{E}_{L} \mathcal{M} E_{R}
\end{aligned}
$$

where $E=\left(e_{1}, e_{2}, e^{i}\right)^{T}$. Here we take $M_{L}$ and $M_{E}$ real and define $m^{\prime}=h_{E}^{\prime} v, \quad m^{\prime \prime}=h_{E}^{\prime \prime} v, \quad \mu_{E}^{i}=\lambda_{E}^{i} v, \quad \mu_{L}^{i}=\lambda_{L}^{i} v$, where $v$ is the vacuum expectation value of the Higgs field. For our purposes we can set the standard model Yukawa couplings $Y_{e}^{i j}$ to zero. $\mathcal{M}$ is diagonalized using two unitary matrices as follows:

$$
\mathcal{M}_{\text {diag }}=V_{L} \mathcal{M} V_{R}^{\dagger},
$$

where the mass eigenstates, $\tilde{E}=\left(\tilde{e}_{1}, \tilde{e}_{2}, \tilde{e}^{i}\right)$, are given by

$$
\begin{gathered}
\tilde{E}_{R}=V_{R} E_{R}, \\
\tilde{E}_{L}=V_{L} E_{L} .
\end{gathered}
$$

Thus for $i=1,2,3$ the $\tilde{e}^{i}$ are the familiar $e, \mu, \tau$. We derive $V_{R}$ and $V_{L}$ by treating off-diagonal elements in $\mathcal{M}$ as a perturbation. (A similar procedure is discussed in Ref. [7].) More explicitly we assume that $v / M_{E, L} \ll 1$ and that $\left|M_{L}-M_{E}\right| \gg v$ and perform the perturbation theory in the small quantity $v / M_{E, L}$. At the leading nontrivial order of perturbation theory,

$$
\begin{gathered}
V_{R}=\left(\begin{array}{ccc}
1 & -p_{R} & q_{R}^{i} \\
p_{R}^{*} & 1 & 0_{1 \times 3} \\
-q_{R}^{* i} & 0_{3 \times 1} & 1_{3 \times 3}
\end{array}\right), \\
V_{L}=\left(\begin{array}{ccc}
1 & -p_{L} & 0_{1 \times 3} \\
p_{L}^{*} & 1 & q_{L}^{* i} \\
0_{3 \times 1} & -q_{L}^{i} & 1_{3 \times 3}
\end{array}\right),
\end{gathered}
$$

where

$$
\begin{array}{ll}
p_{R}=\frac{M_{L} m^{\prime}+M_{E} m^{\prime \prime}}{M_{E}^{2}-M_{L}^{2}}, & q_{R}^{i}=\frac{\mu_{E}^{i}}{M_{L}}, \\
p_{L}=\frac{M_{L} m^{\prime \prime}+M_{E} m^{\prime}}{M_{E}^{2}-M_{L}^{2}}, & q_{L}^{i}=\frac{\mu_{L}^{i}}{M_{E}} .
\end{array}
$$

In the neutrino sector, on the other hand, the heavy neutrino $\nu_{1}$ and linear combinations of ordinary neutrinos are mass eigenstates.

We will perform calculations of loop-level processes in $R_{\xi}$ gauge with $\xi=1$ where not only the contribution from the Higgs boson $h$ but also the fictitious scalar $\phi^{0}$ and $\phi^{+}$ (i.e., the neutral and charged Goldstone boson) must be included. Using Eqs. (9)-(12) we find that their Yukawa couplings in the mass eigenstate basis are (to the order we require)

$$
\begin{aligned}
\mathcal{L}_{\text {Yukawa }}= & \frac{h}{\sqrt{2}}\left[-\lambda_{E}^{i} \overline{\tilde{e}}_{1 L} \tilde{e}_{R}^{i}+\left(h_{E}^{\prime \prime *} q_{R}^{i}-\lambda_{E}^{i} p_{L}^{*}\right) \overline{\tilde{e}}_{2 L} \tilde{e}_{R}^{i}\right. \\
& \left.+\left(h_{E}^{\prime \prime} q_{L}^{i *}+\lambda_{L}^{i *} p_{R}\right) \overline{\tilde{e}}_{1 R} \tilde{e}_{L}^{i}-\lambda_{L}^{i *} \overline{\tilde{e}}_{2 R} \tilde{e}_{L}^{i}\right] \\
& +i \frac{\phi^{0}}{\sqrt{2}}\left[-\lambda_{E}^{i} \overline{\tilde{e}}_{1 L} \tilde{e}_{R}^{i}-\left(h_{E}^{\prime \prime *} q_{R}^{i}+\lambda_{E}^{i} p_{L}^{*}\right) \overline{\tilde{e}}_{2 L} \tilde{e}_{R}^{i}\right. \\
& \left.+\left(h_{E}^{\prime \prime} q_{L}^{i *}-\lambda_{L}^{i *} p_{R}\right) \overline{\tilde{e}}_{1 R} \tilde{e}_{L}^{i}+\lambda_{L}^{i *} \overline{\tilde{e}}_{2 R} \tilde{e}_{L}^{i}\right] \\
& +\phi^{+}\left[-\lambda_{E}^{i} \bar{\nu}_{1 L} \tilde{e}_{R}^{i}+h_{E}^{\prime \prime} q_{L}^{i *} \bar{\nu}_{1 R} \tilde{e}_{L}^{i}\right]+\text { H.c. }
\end{aligned}
$$

The flavor changing couplings of the $Z$ boson to the light charged leptons is important for the calculation of $\mu \rightarrow 3 e$. The relevant terms are

$$
\begin{aligned}
\mathcal{L}_{Z}= & -\frac{g_{Z}}{2} Z_{\mu}\left[q_{R}^{i *} q_{R}^{j} \overline{\tilde{e}}_{R}^{i} \gamma^{\mu} \tilde{e}_{R}^{j}-q_{L}^{i} q_{L}^{j *} \overline{\tilde{e}}_{L}^{i} \gamma^{\mu} \tilde{e}_{L}^{j}\right] \\
& +g_{Z} Z_{\mu}\left[\sin ^{2} \theta_{W} \overline{\tilde{e}}_{R}^{i} \gamma^{\mu} \tilde{e}_{R}^{i}\right. \\
& \left.+\left(-\frac{1}{2}+\sin ^{2} \theta_{W}\right) \overline{\tilde{e}}_{L}^{i} \gamma^{\mu} \tilde{e}_{L}^{i}\right]
\end{aligned}
$$

where $g_{Z}=\sqrt{g_{1}^{2}+g_{2}^{2}}\left[g_{1}\right.$ and $g_{2}$ are gauge coupling constants of $U(1)_{Y}$ and $S U(2)_{L}$, respectively] and $\theta_{W}$ is the Weinberg angle. Integrating out the heavy leptons induces flavor mixing in the $Z$ couplings to the light standard model leptons which causes $\mu \rightarrow 3 e$ at tree level.

\section{RESULTS}

The effective Lagrangian density that describes the impact of integrating out the heavy leptons on the light charged lepton dipole moments and on radiative transitions between light charged lepton flavors is

$$
\mathcal{L}_{\text {eff }}=C_{i j} \overline{\tilde{e}}_{L}^{i} \sigma^{\mu \nu} \tilde{e}_{R}^{j} F_{\mu \nu}+C_{j i}^{*} \overline{\tilde{e}}_{R}^{i} \sigma^{\mu \nu} \tilde{e}_{L}^{j} F_{\mu \nu},
$$

where $\sigma^{\mu \nu}=i\left[\gamma^{\mu}, \gamma^{\nu}\right] / 2$. The coefficients $C_{i j}$ are derived by computing the amplitude for the $\tilde{e}_{R}^{i} \rightarrow \tilde{e}_{L}^{j} \gamma$ process (Fig. 1). We find that

$$
C_{i j}=\frac{e}{64 \pi^{2}}\left[\sum_{B, I} c_{i j}^{B I} \frac{K_{e}\left(M_{I}, m_{B}\right)}{M_{I}}-c_{i j}^{\phi^{+}{ }_{1}} \frac{K_{\nu}\left(M_{1}, M_{W}\right)}{M_{1}}\right],
$$

where $e$ is electric charge, $B=h, \phi^{0}$, and $I=1,2$ (i.e., the index labeling heavy lepton type) and recall that $M_{1} \simeq M_{L}$ and $M_{2} \simeq M_{E}$.

The coefficients $c_{i j}^{B I}$ are

$$
\begin{gathered}
c_{i j}^{h 1}=\frac{1}{2}\left(h_{E}^{\prime \prime *} q_{L}^{i}+\lambda_{L}^{i} p_{R}^{*}\right) \lambda_{E}^{j}, \\
c_{i j}^{\phi^{0} 1}=\frac{1}{2}\left(h_{E}^{\prime \prime *} q_{L}^{i}-\lambda_{L}^{i} p_{R}^{*}\right) \lambda_{E}^{j},
\end{gathered}
$$

for $h-\tilde{e}_{1}$ and $\phi^{0}-\tilde{e}_{1}$ loop diagrams, and

$$
c_{i j}^{h 2}=\frac{1}{2} \lambda_{L}^{i}\left(h_{E}^{\prime \prime *} q_{R}^{j}-\lambda_{E}^{j} p_{L}^{*}\right)
$$



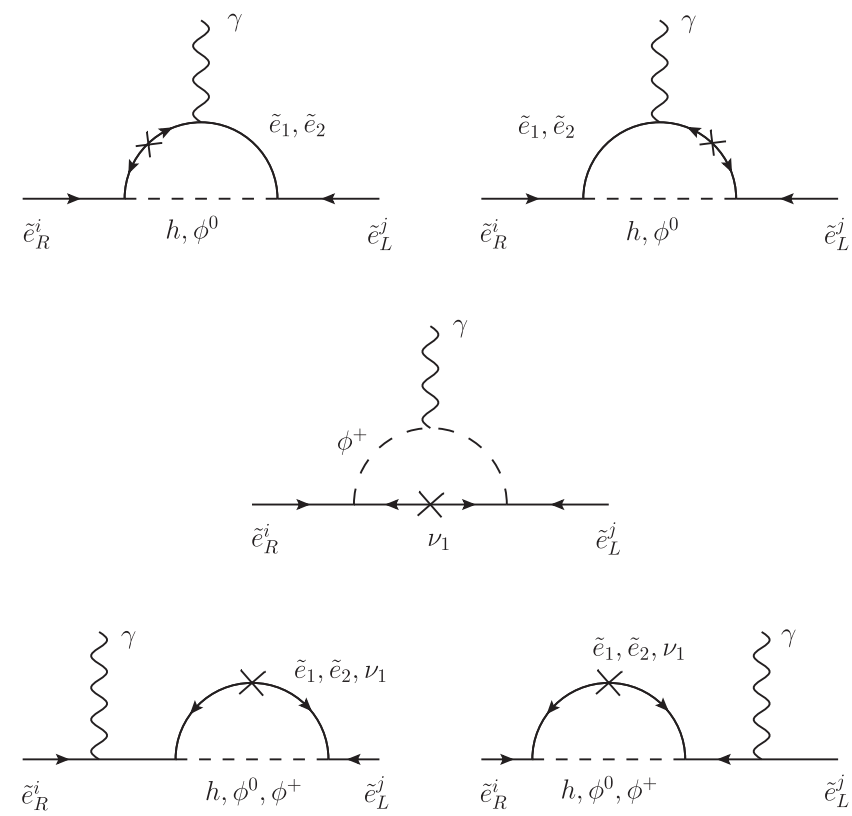

FIG. 1. Diagram for the $\tilde{e}_{R}^{i} \rightarrow \tilde{e}_{L}^{j} \gamma$. Here arrows show the chirality of fermions, and chirality flip is indicated by a cross.

$$
c_{i j}^{\phi^{0} 2}=\frac{1}{2} \lambda_{L}^{i}\left(h_{E}^{\prime \prime *} q_{R}^{j}+\lambda_{E}^{j} p_{L}^{*}\right),
$$

for $h-\tilde{e}_{2}$ and $\phi^{0}-\tilde{e}_{2}$ loop diagrams, and

$$
c_{i j}^{\phi^{+} 1}=h_{E}^{/ / *} q_{L}^{i} \lambda_{E}^{j}
$$

for $\phi^{+}-\nu_{1}$ loop diagrams. $K_{e}\left(M_{I}, m_{B}\right)$ and $K_{\nu}\left(M_{1}, M_{W}\right)$ are loop functions given by

$$
\begin{gathered}
K_{e}\left(M_{I}, m_{B}\right)=\frac{1}{(1-r)^{3}}\left[(1-r)(1-3 r)-2 r^{2} \log r\right], \\
K_{\nu}\left(M_{1}, M_{W}\right)=\frac{1}{(1-r)^{3}}\left[1-r^{2}+2 r \log r\right],
\end{gathered}
$$

where $r=m_{B}^{2} / M_{I}^{2}$ and $r=M_{W}^{2} / M_{1}^{2}$ respectively. $K_{e, \nu} \simeq 1$ when $r \ll 1$. Since we computed in the $R_{\xi}$ gauge with $\xi=1$ the appropriate mass for the fictitious scalars are $m_{\phi^{0}}=M_{Z}$ and $m_{\phi^{+}}=M_{W}$. In this gauge the contribution from the $Z$ and $W$ bosons are negligible. For numerical results, we will take $m_{h}=125 \mathrm{GeV}[16,17]$. In the region where $M_{L, E}$ are multi-TeV, and the Wilson coefficients $C_{i j}$ are given by the very simple expression

$$
C_{i j} \simeq \frac{e \lambda_{L}^{i} \lambda_{E}^{j} m^{\prime \prime *}}{64 \pi^{2} M_{E} M_{L}}
$$

Here we have neglected contributions to the Wilson coefficients $C_{i j}$ that are suppressed by factors of the light lepton masses.

The process $\tilde{e}^{i} \rightarrow \tilde{e}^{j} \tilde{e}^{k} \overline{\tilde{e}}^{k}$ is induced at tree level (Fig. 2). The decay rate of this process is given by (assuming $m_{i} \gg m_{j}, m_{k}$ )

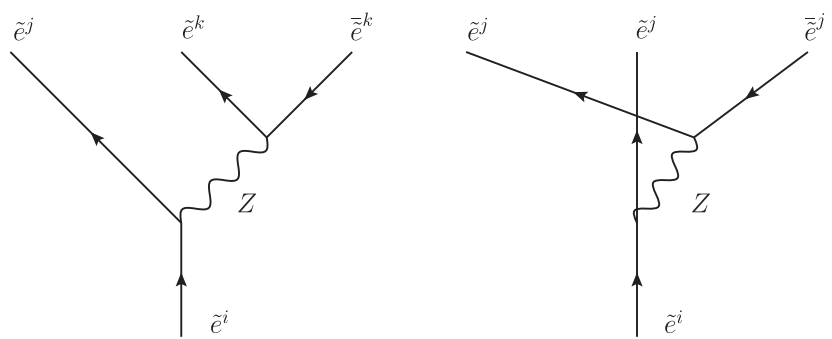

FIG. 2. Diagram of $\tilde{e}^{i} \rightarrow \tilde{e}^{j} \tilde{e}^{k} \overline{\tilde{e}}^{k}$ process. When $k \neq j$, only the left diagram is relevant; meanwhile both diagrams contribute when $k=j$. The crossed diagram has momentum and spin labels interchanged.

$$
\begin{aligned}
\Gamma\left(\tilde{e}^{i} \rightarrow \tilde{e}^{j} \tilde{e}^{j} \overline{\tilde{e}}^{j}\right)= & \frac{m_{i}^{5}}{1536 \pi^{3}}\left[\left|\frac{\lambda_{E}^{i} \lambda_{E}^{j}}{M_{L}^{2}}\right|^{2}\left(\kappa_{L}^{2}+2 \kappa_{R}^{2}\right)\right. \\
& \left.+\left|\frac{\lambda_{L}^{i} \lambda_{L}^{j}}{M_{E}^{2}}\right|^{2}\left(2 \kappa_{L}^{2}+\kappa_{R}^{2}\right)\right],
\end{aligned}
$$

for $k=j$, where $\kappa_{L}=-1 / 2+\sin ^{2} \theta_{W}, \kappa_{R}=\sin ^{2} \theta_{W}$, and

$\Gamma\left(\tilde{e}^{i} \rightarrow \tilde{e}^{j} \tilde{e}^{k} \overline{\tilde{e}}^{k}\right)=\frac{\left(\kappa_{L}^{2}+\kappa_{R}^{2}\right) m_{i}^{5}}{1536 \pi^{3}}\left[\left|\frac{\lambda_{E}^{i} \lambda_{E}^{j}}{M_{L}^{2}}\right|^{2}+\left|\frac{\lambda_{L}^{i} \lambda_{L}^{j}}{M_{E}^{2}}\right|^{2}\right]$,

for $j \neq k$.

The radiative processes which we are interested in are obtained from the Wilson coefficients $C_{i j}$. The decay rate for $\tilde{e}^{i} \rightarrow \tilde{e}^{j} \gamma$ is given by

$$
\Gamma\left(\tilde{e}^{i} \rightarrow \tilde{e}^{j} \gamma\right)=\frac{1}{4 \pi}\left(\left|C_{i j}\right|^{2}+\left|C_{j i}\right|^{2}\right) m_{i}^{3},
$$

where $m_{i}$ is the mass of $\tilde{e}^{i}$ and we have assumed $m_{i} \gg m_{j}$.

EDMs $d_{i}$ of the charged leptons, on the other hand, are coefficients of the effective Lagrangian

$$
\mathcal{L}_{\mathrm{EDM}}=-\frac{i}{2} d_{i} \overline{\tilde{e}}^{i} \sigma^{\mu \nu} \gamma_{5} \tilde{e}^{i} F_{\mu \nu}
$$

and so $d_{i}=-2 \operatorname{Im}\left(C_{i i}\right)$. For later discussion we also give anomalous magnetic dipole moments (MDM) $a_{i}$ of the charged leptons, which are similarly given by

$$
\mathcal{L}_{\mathrm{MDM}}=\frac{e}{4 m_{i}} a_{i} \overline{\tilde{e}}^{i} \sigma^{\mu \nu} \tilde{e}^{i} F_{\mu \nu}
$$

implying that $a_{i}=\left(4 m_{i} / e\right) \operatorname{Re}\left(C_{i i}\right)$.

To summarize, we find that

$$
\begin{gathered}
\Gamma\left(\tilde{e}^{i} \rightarrow \tilde{e}^{j} \gamma\right)=\frac{\alpha v^{2} m_{i}^{3}\left|h_{E}^{\prime \prime}\right|^{2}\left(\left|\lambda_{L}^{i} \lambda_{E}^{j}\right|^{2}+\left|\lambda_{L}^{j} \lambda_{E}^{i}\right|^{2}\right)}{4096 \pi^{4} M_{E}^{2} M_{L}^{2}}, \\
d_{i}=-\frac{e v}{32 \pi^{2}} \frac{\operatorname{Im}\left(h_{E}^{\prime \prime *} \lambda_{L}^{i} \lambda_{E}^{i}\right)}{M_{E} M_{L}},
\end{gathered}
$$




$$
a_{i}=\frac{m_{i} v}{16 \pi^{2}} \frac{\operatorname{Re}\left(h_{E}^{\prime \prime *} \lambda_{L}^{i} \lambda_{E}^{i}\right)}{M_{E} M_{L}} .
$$

Equations (27), (28), and (32)-(34) are the principal analytic results of this paper. They are valid in the limit that $v / M_{L, E} \ll 1$ and $m_{i} / v \ll 1$.

Plugging in the values of the standard model parameters and focusing on the quantities $\operatorname{Br}(\mu \rightarrow e \gamma), \operatorname{Br}(\mu \rightarrow 3 e)$, $\left|d_{e}\right|$, and $\left|a_{\mu}\right|$ the above implies that

$$
\begin{aligned}
& \operatorname{Br}(\mu \rightarrow e \gamma) \simeq 2.3 \times 10^{-12}\left|\frac{h_{E}^{\prime \prime}}{10^{-4}}\right|^{2}\left(\frac{10^{2} \mathrm{TeV}^{2}}{M_{E} M_{L}}\right)^{2} \\
& \times\left(\left|\lambda_{L}^{\mu} \lambda_{E}^{e}\right|^{2}+\left|\lambda_{L}^{e} \lambda_{E}^{\mu}\right|^{2}\right), \\
& \operatorname{Br}(\mu \rightarrow 3 e) \simeq 1.7 \times 10^{-8} \times\left[\left|\lambda_{E}^{e} \lambda_{E}^{\mu} \frac{10^{2} \mathrm{TeV}^{2}}{M_{L}^{2}}\right|^{2}\right. \\
&\left.+1.1 \times\left|\lambda_{L}^{e} \lambda_{L}^{\mu} \frac{10^{2} \mathrm{TeV}^{2}}{M_{E}^{2}}\right|^{2}\right], \\
&\left|d_{e}\right| \simeq 1.1 \times 10^{-26} e \mathrm{~cm}\left|\operatorname{Im}\left(\frac{h_{E}^{\prime *} \lambda_{L}^{e} \lambda_{E}^{e}}{10^{-4}}\right)\right|\left(\frac{10^{2} \mathrm{TeV}^{2}}{M_{E} M_{L}}\right),
\end{aligned}
$$

and

$$
\left|a_{\mu}\right| \simeq 1.2 \times 10^{-13}\left|\operatorname{Re}\left(\frac{h_{E}^{\prime \prime *} \lambda_{L}^{\mu} \lambda_{E}^{\mu}}{10^{-4}}\right)\right|\left(\frac{10^{2} \mathrm{TeV}^{2}}{M_{E} M_{L}}\right) .
$$

Equations (35)-(38) are compared with the measured values given in Eqs. (4)-(6) and the deviation of the observed muon $g-2$ [18] from its standard model prediction [19],

$$
\Delta a_{\mu}^{\exp }=a_{\mu}^{\exp }-a_{\mu}^{\mathrm{SM}} \simeq(2.8 \pm 0.8) \times 10^{-9} .
$$

Note that while it is expected that a $10 \mathrm{TeV}$ vectorlike lepton can give an observable contribution to $\mu \rightarrow e \gamma$, $\mu \rightarrow 3 e$, and the electron EDM, such a heavy vectorlike lepton does not give a large enough contribution to the anomalous magnetic moment to explain the discrepancy between standard model value and its observed value.

Before going on to the numerical results, we comment on the perturbative corrections to a light lepton mass matrix that come from integrating out the heavy leptons. There is a tree-level correction to light lepton mass matrix elements, which is induced by mixing with heavy leptons. It is given by

$$
\left|\Delta m_{i j}^{\text {tree }}\right|=\left|m^{\prime \prime} q_{R}^{i} q_{L}^{j}\right| .
$$

This result is consistent with Ref. [6]. In addition to this correction, there is also a logarithmically divergent oneloop correction to the light lepton mass matrix that arises from one-loop self-energy diagrams analogous to the Feynman diagrams that contribute to the radiative transitions and dipole moments but without the photon. Using a momentum cutoff $\Lambda$, it is

$$
\begin{aligned}
\Delta m_{i j}^{\text {loop }} & =-\frac{1}{16 \pi^{2}} \sum_{B, I} c_{i j}^{B I} M_{I} \log \left(\frac{\Lambda^{2}}{M_{I}^{2}}\right) \\
& \simeq-\frac{\lambda_{L}^{i} \lambda_{E}^{j} m^{\prime \prime *}}{16 \pi^{2}}\left[\frac{2 M_{L}}{M_{E}} \log \left(\frac{\Lambda^{2}}{M_{L}^{2}}\right)+\frac{M_{E}}{M_{L}} \log \left(\frac{\Lambda^{2}}{M_{E}^{2}}\right)\right],
\end{aligned}
$$

assuming $\Lambda \gg M_{I} \gg m_{h}, m_{Z}$. This quantum correction is more important than the tree-level one when $M_{L, E}$ is greater than about a few hundred $\mathrm{GeV}$. In the region of heavy vectorlike leptons masses we are focusing on the fact that the tree-level contribution from mixing with the heavy leptons can be neglected and so in order to avoid awkward cancellations between $\Delta m_{i j}=\Delta m_{i j}^{\text {tree }}+$ $\Delta m_{i j}^{\text {loop }} \simeq \Delta m_{i j}^{\text {loop }}$ and the standard model tree-level light lepton mass matrix elements from Eq. (2) (which are proportional to $Y_{e}^{i j}$ ), we adopt the naturalness criterion [20]:

$$
\left|\Delta m_{i j} \Delta m_{j i}\right| \lesssim m_{i} m_{j} .
$$

A more precise analysis of naturalness constraints would involve solving renormalization group equations for the evolution of the coupling constants in the theory.

It is important to remember that the region of parameter space excluded by Eq. (42) is not ruled out and can be consistent with experiment. We view the region of parameter space that does not satisfy Eq. (42) as disfavored by the naturalness criteria that the one-loop correction to the light lepton mass matrix calculated with a large momentum space cutoff not overwhelm the contribution which is coming from Eq. (2).

We have computed the parts of the amplitudes for $\mu \rightarrow e \gamma$ and the electric dipole moment of the electron that are not suppressed by the light lepton masses. The radiative correction to the light lepton mass matrix in Eq. (41) is proportional to the same combination of couplings constants that occur in the amplitudes for these radiative processes. Hence the naturalness condition in Eq. (42) restricts the size of this part of their amplitudes. There are situations where the radiative correction to the charged lepton mass matrix is negligible and the rate for $\mu \rightarrow 3 e$ not suppressed, for example, if $h_{E}^{\prime \prime}$ is extremely small but $\lambda_{E, L}$ order unity. In this case the contributions to the amplitudes for the radiative processes proportional to the light lepton masses, that we have not calculated, dominate. However, in that situation the rate for $\mu \rightarrow 3 e$ is sensitive to higher mass scales for the vectorlike leptons than the rate for $\mu \rightarrow e \gamma$ since $\operatorname{Br}(\mu \rightarrow e \gamma) \sim$ $(\alpha / 4 \pi) \operatorname{Br}(\mu \rightarrow 3 e)$.

\section{NUMERICAL RESULTS}

First we consider case (i) discussed in Sec. II. We take $h_{E}^{\prime}=h_{E}^{\prime \prime}=h=10^{-3}, \quad \lambda_{E}^{i}=\lambda_{L}^{i}=\lambda$, and $M_{E}=$ $1.5 M_{L}$. Figure 3 shows contours of fixed $\operatorname{Br}(\mu \rightarrow e \gamma)$, 

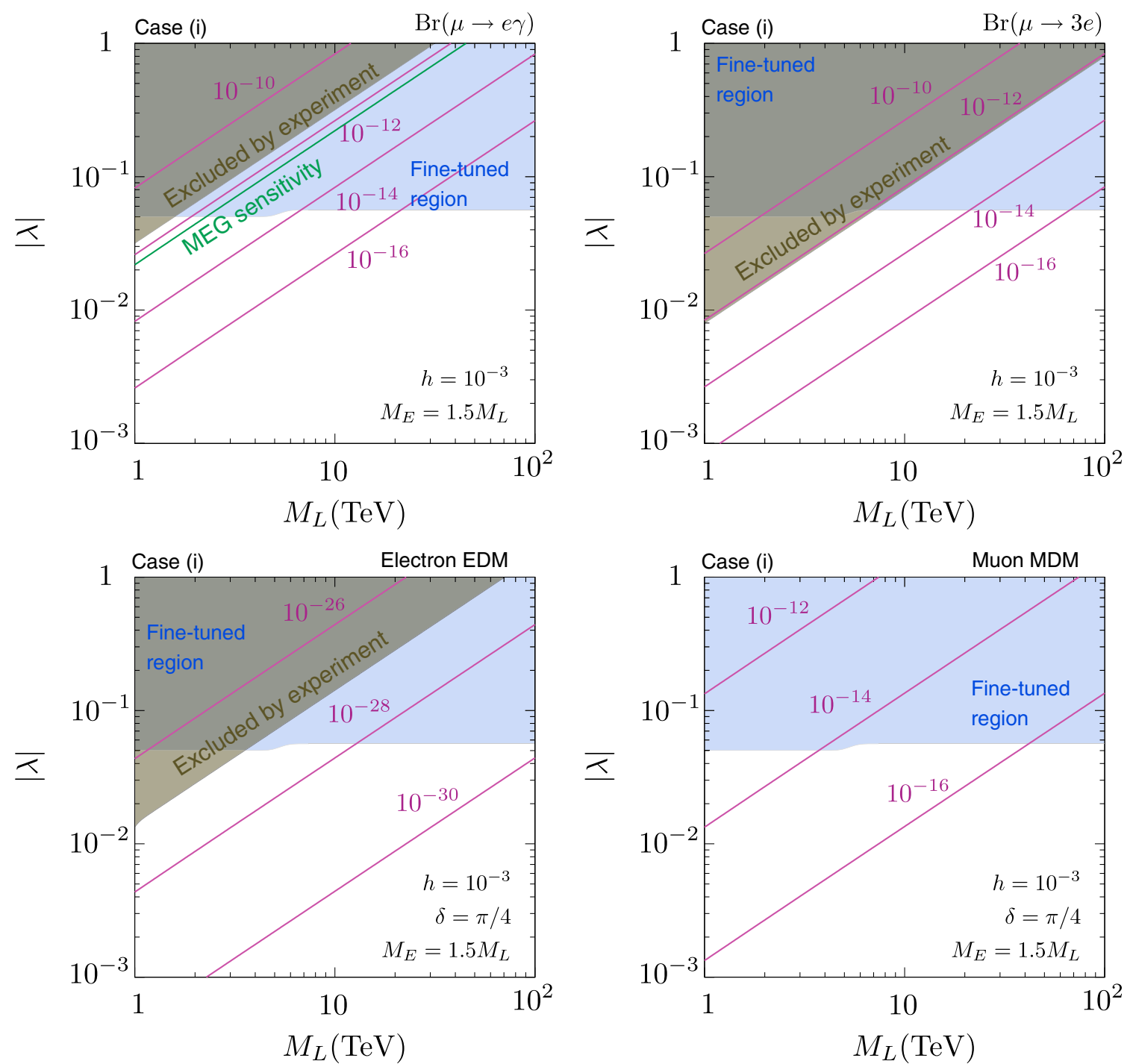

FIG. 3 (color online). Contours of fixed $\operatorname{Br}(\mu \rightarrow e \gamma)$ (top left), $\operatorname{Br}(\mu \rightarrow 3 e)$ (top right), electron EDM (bottom left), and muon MDM (bottom right) in the $M_{L^{-}}|\lambda|$ plane for the parameters discussed in the text. Lines $\operatorname{are} \operatorname{Br}(\mu \rightarrow e \gamma)=\operatorname{Br}(\mu \rightarrow 3 e)=10^{-10}$, $10^{-12}, 10^{-14}, 10^{-16},\left|d_{e}\right|=10^{-26}, 10^{-28}, 10^{-30} e \mathrm{~cm}$, and $\left|a_{\mu}\right|=10^{-12}, 10^{-14}, 10^{-16}$ from top to bottom. The dark-shaded region is excluded by experiments, while light-shaded (blue) indicates fine-tuned parameter space. In the top left, the prospective MEG sensitivity is also plotted.

$\operatorname{Br}(\mu \rightarrow 3 e)$, electron EDM, and muon MDM in the $M_{L^{-}}|\lambda|$ plane. The dark shaded region is excluded by experiments. We also shade the fine-tuned parameter space where Eq. (42) is not satisfied, taking $\Lambda=10^{15} \mathrm{GeV}$. Note that, $\mu \rightarrow 3 e$ gives a more stringent constraint on the parameter space. This is because $\operatorname{Br}(\mu \rightarrow 3 e)$ is not suppressed by $h$. The current experimental bound constrains $\left|h \lambda^{2}\right| \lesssim 10^{-5}$ when $M_{L} \sim 10 \mathrm{TeV}$. On the other hand, the region of parameter space where $\left|h \lambda^{2}\right| \geqslant 10^{-5}$ is fine tuned. For the electron EDM, we take $\delta=\pi / 4$ where $\arg \left(\lambda^{2}\right)=\delta$. The region of the plane excluded by experiment is almost the same as in the $\mu \rightarrow 3 e$ case. Note that the rate for $\mu \rightarrow 3 e$ does not depend on $h$ and if we take $h$ much smaller then the fine-tuned region disappears.
There are also experimental constraints on flavor changing $\tau$ lepton decays. Since the weak decay width of a charged lepton of type $i$ is proportional to $m_{i}^{5}$, the branching fractions for $\tau \rightarrow \mu \gamma$ and $\tau \rightarrow e \gamma$ satisfy $\operatorname{Br}(\tau \rightarrow \mu \gamma) \simeq \operatorname{Br}(\tau \rightarrow e \gamma) \simeq\left(m_{\mu} / m_{\tau}\right)^{2} \operatorname{Br}(\mu \rightarrow e \gamma) \quad$ in case (i). Current experimental bounds for these process are by $\operatorname{Br}(\tau \rightarrow \mu \gamma)<4.4 \times 10^{-8}$ and $\operatorname{Br}(\tau \rightarrow e \gamma)<$ $3.3 \times 10^{-8}$ [21]. Thus these bounds are less stringent than for muon decays.

For reference, we have given the contours of fixed muon MDM. Clearly the impact of vectorlike leptons on the muon MDM is negligible in the parameter region on which we have focused. In order to explain the muon $g-2$ anomaly by vectorlike leptons, they should be lighter 

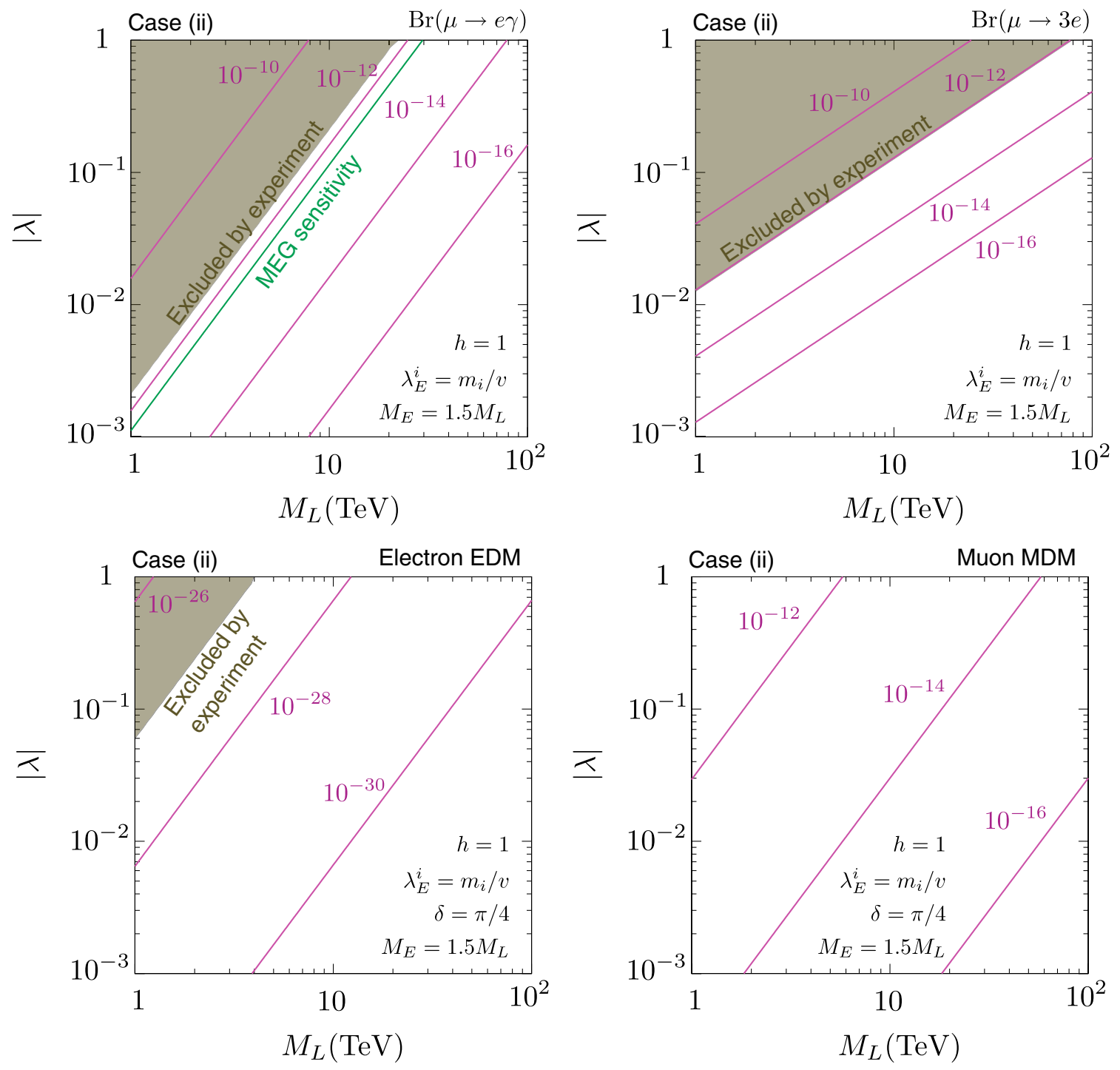

FIG. 4 (color online). Same as Fig. 3 but with different choice of parameters.

than $1 \mathrm{TeV}$, for example, $M_{L, E} \sim O(100 \mathrm{GeV})$ with $\left|h \lambda^{2} \cos \delta\right| \sim 10^{-3}$. Note that having vectorlike leptons with masses around the weak scale does not necessarily lead to a conflict with the limits on the rates for $\mu \rightarrow e \gamma$, $\mu \rightarrow 3 e$, and the electron electric dipole moment. For example, it could be the case that $\left|\lambda_{E, L}^{e}\right|$ is much smaller than $\left|\lambda_{E, L}^{\mu}\right|$.

Next we consider the case (ii). [Parametrically case (iii) is identical to case (ii).] We take $h_{E}^{\prime}=h_{E}^{\prime \prime}=h=1, \lambda_{L}^{e}=$ $\lambda_{L}^{\mu}=\lambda, \lambda_{E}^{i}=m_{i} / v$, and $M_{E}=1.5 M_{L}$. Figure 4 shows the results. [For the electron EDM, we take $\delta=\arg (\lambda)=$ $\pi / 4$.] In this case there is no fine-tuned region (taking $\Lambda=$ $10^{15} \mathrm{GeV}$ ). Since the electron EDM is suppressed by $\left|\lambda_{E}^{e}\right|$, the experimental bound becomes weaker compared to the previous case. The current bounds from the limits on the $\mu \rightarrow e \gamma$ and $\mu \rightarrow 3 e$ branching ratios, on the other hand, give a more severe constraint on the parameter space. For $\mu \rightarrow e \gamma$, this is because the branching ratio is only suppressed by $\left|\lambda_{E}^{\mu}\right|$. The numerical result indicates that the $M_{E, L} \sim 10-100 \mathrm{TeV}$ region may be probed in the future MEG experiment, which has prospective sensitivity of $\operatorname{Br}(\mu \rightarrow e \gamma) \simeq 5.0 \times 10^{-13}$. [For $\tau$ radiative decay, we note that $\operatorname{Br}(\tau \rightarrow \mu \gamma)=\operatorname{Br}(\mu \rightarrow e \gamma)$ in this case.] The process $\mu \rightarrow 3 e$, on the other hand, is not suppressed by any of the $\left|\lambda_{E}^{i}\right|$ [see Eq. (36)], which gives rise to a large branching fraction. It is especially interesting that the $M_{L, E} \sim 100 \mathrm{TeV}$ region with $|\lambda| \sim O(1)$ is already excluded by current experiments, which means vectorlike leptons with masses up to $100 \mathrm{TeV}$ can have an observable impact on experiments.

\section{CONCLUDING REMARKS}

We have considered the phenomenological impact of vectorlike leptons that are much heavier than the weak scale. We discussed expectations for their couplings based 
on the observed masses of the charged leptons. With couplings that are reasonable from this perspective we found that vectorlike leptons with masses up to $100 \mathrm{TeV}$ can have an impact on the electric dipole moment of the electron and the rates for $\mu \rightarrow 3 e$ and $\mu \rightarrow e \gamma$ that are detectable in the next generation of experiments. When the vectorlike leptons are this heavy their impact on the anomalous magnetic moment of the muon and Higgs properties is very small.

For the chirality flipping radiative processes $\mu \rightarrow e \gamma$ and the electric dipole moment of the electron, we have focused on the parts of their amplitudes that are not suppressed by the light lepton masses. One of the goals of this work has been to explore whether the absence of light lepton mass suppression results in sensitivity to much higher masses for the vectorlike leptons. We found that one-loop radiative corrections to the light lepton mass matrix are proportional to the same combination of couplings and demanding that the one-loop contribution not overwhelm the contribution from Yukawa couplings restricts the reach in vectorlike lepton mass. Finally we noted that, for similar branching ratios, the three body flavor changing decay $\mu \rightarrow 3 e$ has sensitivity to greater vectorlike lepton masses than the radiative two body decay $\mu \rightarrow e \gamma$.

In this paper we have focused on purely leptonic processes that are free of hadronic uncertainties. Nevertheless it is worth noting that in these models tree-level $Z$ exchange gives rise to an effective Lagrangian that dominates $\mu$ to $e$ conversion in nuclei. Performing a naive matching from quarks to nucleons (see Refs. [22,23]) and dropping terms that are expected to be suppressed, the effective Lagrangian is

$$
\begin{aligned}
\mathcal{L}_{\mu \rightarrow e}= & -2\left[\frac{\lambda_{E}^{e *} \lambda_{E}^{\mu}}{M_{L}^{2}} \bar{e}_{R} \gamma^{\alpha} \mu_{R}-\frac{\lambda_{L}^{e} \lambda_{L}^{\mu *}}{M_{E}^{2}} \bar{e}_{L} \gamma^{\alpha} \mu_{L}\right] \\
& \times\left[\left(\frac{1}{4}-\sin ^{2} \theta_{W}\right) \bar{p} \gamma_{\alpha} p-\frac{1}{4} \bar{n} \gamma_{\alpha} n\right]
\end{aligned}
$$

\section{ACKNOWLEDGMENTS}

This paper is funded by the Gordon and Betty Moore Foundation through Grant No. 776 to the Caltech Moore Center for Theoretical Cosmology and Physics. The work of the authors was supported also in part by the U.S. Department of Energy under Contract No. DE-FG0292ER40701. The research of M. B. W. was supported in part by Perimeter Institute for Theoretical Physics. Research at Perimeter Institute is supported by the Government of Canada and by the Province of Ontario through the Ministry of Economic Development and Innovation.
[1] P. Fileviez Perez and M. B. Wise, J. High Energy Phys. 08 (2011) 068.

[2] K. Ishiwata and M. B. Wise, Phys. Rev. D 84, 055025 (2011).

[3] K. Kannike, M. Raidal, D. M. Straub, and A. Strumia, J. High Energy Phys. 02 (2012) 106; 10 (2012) 136(E).

[4] F. del Aguila, J. de Blas, and M. Perez-Victoria, Phys. Rev. D 78, 013010 (2008).

[5] A. Joglekar, P. Schwaller, and C. E. M. Wagner, J. High Energy Phys. 12 (2012) 064.

[6] J. Kearney, A. Pierce, and N. Weiner, Phys. Rev. D 86, 113005 (2012).

[7] R. Dermisek and A. Raval, Phys. Rev. D 88, 013017 (2013).

[8] R. Harnik, J. Kopp, and J. Zupan, J. High Energy Phys. 03 (2013) 026.

[9] D. McKeen, M. Pospelov, and A. Ritz, Phys. Rev. D 86, 113004 (2012).

[10] F. del Aguila and M. J. Bowick, Phys. Lett. 119B, 144 (1982).

[11] F. del Aguila and M. J. Bowick, Nucl. Phys. B224, 107 (1983).

[12] J. M. Arnold, B. Fornal, and M. B. Wise, Phys. Rev. D 88, 035009 (2013).
[13] J. Adam et al. (MEG Collaboration), Phys. Rev. Lett. 107, 171801 (2011).

[14] U. Bellgardt et al. (SINDRUM Collaboration), Nucl. Phys. B299, 1 (1988).

[15] J. J. Hudson, D. M. Kara, I. J. Smallman, B. E. Sauer, M. R. Tarbutt, and E. A. Hinds, Nature (London) 473, 493 (2011).

[16] G. Aad et al. (ATLAS Collaboration), Phys. Lett. B 716, 1 (2012).

[17] S. Chatrchyan et al. (CMS Collaboration), Phys. Lett. B 716, 30 (2012).

[18] G. W. Bennett et al. (Muon G-2 Collaboration), Phys. Rev. D 73, 072003 (2006).

[19] M. Davier, A. Hoecker, B. Malaescu, and Z. Zhang, Eur. Phys. J. C 71, 1515 (2011); 72, 1874(E) (2012).

[20] T.P. Cheng and M. Sher, Phys. Rev. D 35, 3484 (1987).

[21] B. Aubert et al. (BABAR Collaboration), Phys. Rev. Lett. 104, 021802 (2010).

[22] R. Kitano, M. Koike, and Y. Okada, Phys. Rev. D 66, 096002 (2002); 76, 059902(E) (2007).

[23] V. Cirigliano, R. Kitano, Y. Okada, and P. Tuzon, Phys. Rev. D 80, 013002 (2009). 